\title{
Demarcation of the Vibration Markers based on Gradient Information HT Method
}

\author{
Dai Fei ${ }^{1, a}$, Wang Yuzhen ${ }^{1, a}$, Xin Mai ${ }^{2, b}$, Li Yalin ${ }^{3, c}$ \\ ${ }^{1}$ Lanzhou General Hospital, Lanzhou Military Area Command, Gansu, Lanzhou 730050, China \\ ${ }^{2}$ ShengNeng Electricity Engineering Co, LTD. of MeiShan, MeiShan, SiChuan, 620010, China \\ ${ }^{3}$ School of Foreign Language, Lanzhou University of Technology, Gansu, Lanzhou 730050,China \\ a369830863@qq.com, bjiangwei_xm@aliyun.com, ${ }^{\mathrm{c}}$ liyalin-001@163.com
}

Keywords: Centers of vibration markers; MATLAB; HT method

\begin{abstract}
Purpose: We do it for tracking the markers of experiments on vibration platform. Method: We recommended HT method which owns better robustness, then designed the programs by the wa $\mathrm{y}$ of MATLAB to demarcate the centers of vibration markers. Result: We have successfully demarc ated the centers of vibration markers. Conclusion: Using the method described above can realize ex pectations.
\end{abstract}

\section{Introduction.}

Photo-grammetry[1] is a kind of global, non-contact method used in many fields[2], which is wit $\mathrm{h}$ the feature of high degree of automation and environmental adaptable. This method can also help get the data as speed, acceleration and trajectory while we are recording the time signal. Using mark ers to assist researching on building deformation for vibration platform is considered to be an impor tant method to improve the accuracy and speed[3].

Hough Transform is an efficacious method to research on brink of figures[4], which owns good i mmunity and can work on several ones at the same time[5]. In this way, we'll easily mark the vibrat ion platform for the following research.

\section{The building of measuring system}

We used digital camera (HDR-SR12, SONY) as the collector, which owns max sampling frequen cy of $800 \mathrm{fm} / \mathrm{s}$ to satisfy technical requirements.

Form 1. The parameters of SONY HDR-SR12 digital camera

\begin{tabular}{l|l}
\hline Parameters Model/Data & Parameters Model/Data \\
\hline Sensor CMOS & Camera Features $1920 \times 1080$ \\
Size of image 96dpi & Speed of Shutter 1/25-1/800 \\
Size of storage MPEG, JPEG & Ram 120G \\
\hline
\end{tabular}

As fig. 1 shows, you can notice vibration markers. In this experiment, we employed manual mea surement method, which can meet the demand for its 0.5 pixel in accuracy[6].The steps: first, we $g$ ot the edge image of markers by using Grade edge detection operator, such as Fig. 1(b) shows; and $t$ hen, we used HT method to precisely mark the location of markers in each picture, which is the key step of the method.

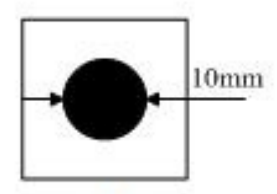

(a)

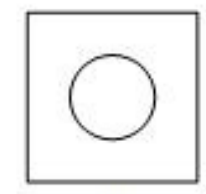

(b)

Fig.1. marker and edge image

The algorithm process of HT method is shown as Fig.2 


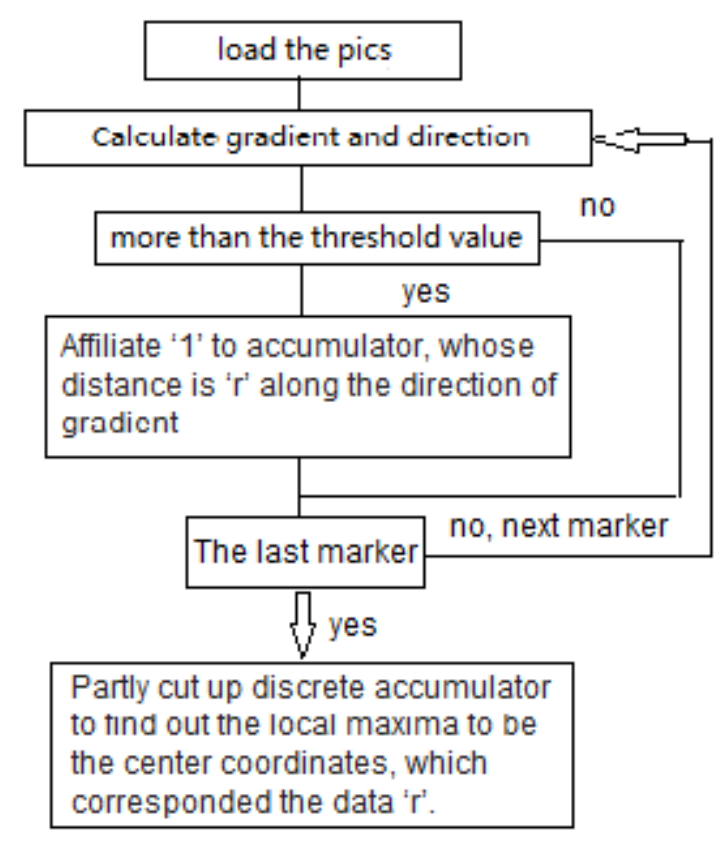

Fig.2. the algorithm process of HT method clc

We realized the algorithm above by using 'MATLAB', whose program is shown as following.

close all;

clear

bw=imread('F: $\backslash$ research.jpg');

$\mathrm{f}=\mathrm{rgb} 2 \mathrm{gray}(\mathrm{bw})$;

$\mathrm{g}=\mathrm{im} 2 \mathrm{bw}(\mathrm{f}, .5)$;

$\mathrm{bw}=$ medfilt2 $(\sim \mathrm{g},[55])$;

imshow(bw)

hold on;

[c,r]=imfindcircles(bw,[10,1000],'Method','PhaseCode','Sensitivity',.95,'EdgeThreshold',.96); viscircles(c,r);

for $i=1$ :length(r)

plot(c(i,1),c(i,2),'ko'); title('HT method to mark the center')\%the center end

\section{Application and Analysis}

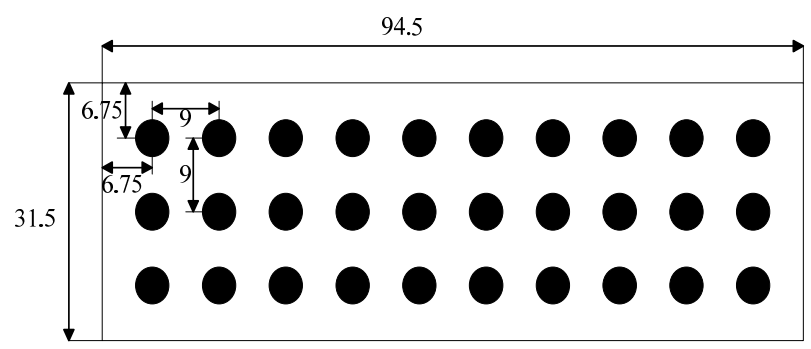

Fig.3. The original image with precise coordinate (Measurement: $\mathrm{mm}$ )

As you see above, Fig.3 shows the markers with precise coordinate. used HT method based on Gradient Information to mark the centers, then the result is shown in Fig.4 (below). 

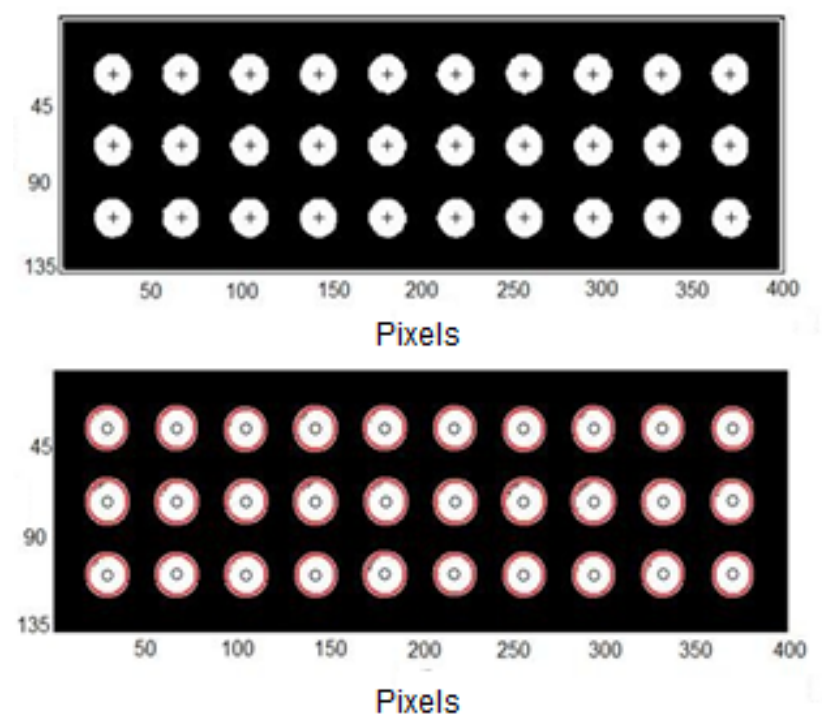

Fig.4. The actual centers (above) and the centers processed by HT method (below)

The coordinates of the markers shown in Fig.4 (above) were worked out by 'MATLAB' metho d. And the coordinates of the markers shown in Fig.4 (below) were worked out by HT method. Fig. 5 shows the result of the contradistinction, which was programmed by 'MATLAB', to certify the a ccuracy of HT method.

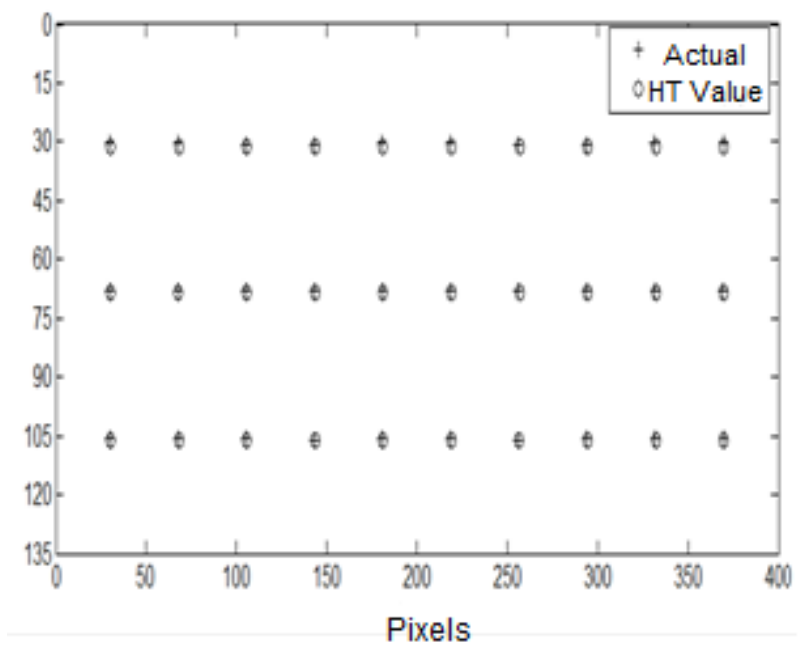

Fig.5. The comparison on result between HT method and the actual

In Fig.5, '+' shown the actual coordinates as Fig.4 (above) and 'o' shown the coordinates work ed out by HT method as Fig.4 (below).We noticed using HT method can help accurately mark the vibration markers by the comparison

\section{Conclusion}

It is proved that, this method can realize the design we made before, apart from this, it owns go od suitability and stability in dealing with the irregular markers, which can be great helpful in rese arching on the deformation of large equipment in future.

\section{Reference}

[1] Yu Yonghua. The Analysis on Photogrammetry and Remote Sensing Technology[J]. Jiangxi B uilding Materials, 2014, 1: 217.

[2] Li Guidong, Liu Zhixing. Direct Linear Tranformation-Based Calibration Method and Accurac y Analysis[J], GEOMATICS \& SPATIAL INFORMATION TECHNOLOGY ,2013.12,36(12): 227 -228 . 
[3] Song Limei, Chen Changman, Chen Zhuo. Detection and recognition of cyclic coded targets[J], Optics and Precision Engineering, 2013.12,21(12): 3239-3247.

[4] Chen Si, Zhao Huichang. Scattering Center Extraction of UWB Radio Fuze's Target Based on Polar Hough Transform[J], Preparedness Technology: English, 2012,2: 74-78.

[5] Xu hua, Li Tianbin, Wang Dong. STUDY OF SEISMIC RESPONSES OF MOUNTAIN TUN NELS WITH 3D SHAKING TABLE MODEL TEST[J], Chines Journal of Rock Mechanics and E ngineering, 2013.9,32 (9) : 1762-1771

[6] Zhang Yongsheng. Remote Imaging System[M]. Beijing: Science Press, 2000, vol.4:38-39,2 005 . 